\title{
Thirty-Fourth Annual AOA Research Conference Abstracts, 1990: Part 1
}

Part 1 of abstracts of papers and poster sessions to be presented at the Thirty-Fourth Annual AOA Research Conference, Nov 25 to 29, includes those in the Burroughs Wellcome, Osteopathic Manipulative Treatment/Osteopathic Principles and Practice, and General Clinical Medicine categories. Abstracts in the remaining categories will appear in the October issue of JAOA.

\section{Burroughs Wellcome}

\section{Cervical range of motion: Can it be improved through the use of osteopathic manipulation?}

RAY BENSON, DO

Ohio University College of Osteopathic Medicine Athens, Ohio

Osteopathic manipulative treatment has been used to restore function in those with musculoskeletal complaints. The purpose of this study is to document muscle energy technique as an effective method of increasing cervical range of motion, thereby improving function, in the patient with decreased cervical mobility with or without associated pain. The study will be completed in two parts. First, a pilot study will commence using six subjects randomly assigned to either an experimental or control group. A technician will measure and record cervical range of motion both prior to and following treatment using a gravity goniometer with spirit level and head adapter. The patients in the experimental group will receive a muscle energy technique treatment. The control group will receive a sham ultrasound treatment. Both groups will be remeasured 3 days following treatment. If no changes in protocol are necessary after the pilot study is completed, the main study will commence with 40 subjects being randomly assigned to either the experimental or control groups. Data will be analyzed using the dependent $t$ test.

Supported by Burroughs Wellcome Osteopathic Research Fellowship No. F-89-04.

Determination of the sensitivity and specificity of prostate specific antigen and transrectal ultrasound of the prostate in detecting stage A prostate cancer prior to prostatectomy

ROBERT L. FIORELLI, DO

ROBERT L. KLAUS, MD

LAURENCE BELKOFF, DO

SAMUEL MANFREY, DO

LEONARD FINKELSTEIN, DO

Osteopathic Medical Center of Philadelphia and

Albert Einstein Medical Center

Philadelphia, Pennsylvania

We prospectively studied 103 men with normal prostate examinations by palpation who were considered to have bladder outlet obstruction secondary to prostatic hypertrophy and who were in need of 
transurethral prostatectomy. All men underwent a preoperative transrectal ultrasound examination of the prostate and prostate specific antigen (PSA) level determination. A total of 30 cancers were ultimately detected and $22(73 \%)$ of these were detected preoperatively by either an abnormality on ultrasound examination or an elevated PSA or both. Eight of these men were spared transurethral prostatectomy and had definitive treatment of their disease based on a transrectal biopsy specimen and appropriate staging evaluation.

When we compared the sensitivities, specificities, and positive/negative predictive values for PSA and ultrasound combined, the sensitivities and negative predictive values for cancer were superior to the specificities and positive predictive values $(92 \%$ and $94 \%$ vs $71 \%$ and $64 \%$, respectively). Because of this, we feel that both studies are useful prior to prostatectomy to rule out cancer. We do not recommend their routine use to screen the general male population older than 40 years, since a large number of unindicated biopsies may need to be performed. However, in patients who are candidates for prostatectomy, their combined use appears to be very sensitive in the early detection of carcinoma.

Supported by Burroughs Wellcome Osteopathic Research Fellowship No. F-89-01.

\section{Naloxon potentiates adrenergic vasoconstriction in isolated arteries from canine skeletal muscle}

\section{SCOTT T. STOLL, BS}

J. L. CAFFREY, PhD

Department of Physiology

Texas College of Osteopathic Medicine

Fort Worth, Texas

Naloxone improves the clinical cardiovascular status in circulatory shock. Naloxone potentiates cardiac responses to infused epinephrine and facilitates the release of norepinephrine from vascular sympathetic nerve terminals. Our study was designed to examine the effects of opiate receptor blockade with naloxone in isolated canine skeletal muscle arteries, independent of neural, humoral, or mechanical factors. Small (1 mm OD) arterial segments were dissected free and suspended horizontally in oxygenated Krebs-Ringer bicarbonate glucose solution at $37^{\circ} \mathrm{C}$. We found that naloxone $\left(10^{5} \mathrm{M}\right)$ potentiates epinephrine-induced vasoconstriction, but has no vasoconstrictive effect when given alone. In the dose range from $10^{6}$ to $3 \times 10^{5}$ $\mathrm{M}$, naloxone potentiated contractions by epinephrine the most, norepinephrine to a lesser degree, and phenylephrine not at all.

These results indicate that naloxone can potentiate catecholamine-induced vasoconstriction independent of central cardiovascular or neural reflex interactions. The response is relatively specific for epinephrine and may involve nontraditional opiate receptors or nonreceptor mechanisms.

Supported by Burroughs Wellcome Osteopathic Research Fellowship No. F-89-03.

\section{Comparison of thoracic pump and incentive spirometry}

\author{
SANDRA LEE OPPENHEIMER, DO \\ Olympia Fields Osteopathic Medical Center \\ Olympia Fields, Illinois
}

Osteopathic manipulative treatment in pulmonary disease has shown its effectiveness in practice, but the experimental evidence to support it is limited. Studying postcholecystectomy atelectasis allowed comparison of the course of disease and osteopathic manipulative treatment (thoracic lymphatic pump [TLP]) and the standard of care (incentive spirometry [IS)]. A randomized, physician-blind research design was used to compare the TLP with IS. The hypothesis tested was that there was no significant difference between TLP and IS in the prevention of postoperative atelectasis. The study was approved by the Chicago Osteopathic Hospital Systems Institutional Review Board. All patients undergoing cholecystectomy during the studied period were included in the analysis. Patients were excluded from the comparison study if a predetermined minimal number of treatments were not received, surgery requiring other than a subcostal incision was performed, or the patient refused or withdrew from the study. Respiratory parameters $\left(\mathrm{FVC}\right.$ and $\mathrm{FEV}_{1}$ ) were measured on each subject preoperatively and daily postoperatively. Each subject received a physical examination and an assessment for atelectasis daily. The TLP and IS treatments were given three times a day. A low incidence of atelectasis occurrence and no significant difference in respiratory parameters between groups was found. In conclusion, the TLC and IS 
were found to be equally effective in the prevention of postoperative atelectasis.

Supported by Burroughs Wellcome Osteopathic Research Fellowship No. F-89-06.

\section{Effects of an educational intervention on quality and frequency of osteopathic structural documentation on hospital admitting examination}

\section{HARRY D. FRIEDMAN, DO}

WILLIAM L. JOHNSTON, DO

ALBERT F. KELSO, PhD

FREDERICK N. SCHWARTZ, DO

Chicago College of Osteopathic Medicine

Chicago, Illinois

The purpose of this study was to (1) develop a standardized instructional videotape on the procedure for documentation of osteopathic findings and diagnosis for use in the hospital setting; (2) and study its influence on the correlation of palpation findings with a diagnostic impression on hospital admitting examinations.

Before and after viewing an educational videotape on recording osteopathic structural findings and impressions, random hospital charts were pulled and the admitting examination was evaluated. The frequency of documentation of altered findings (structure, motion, and/or tissue), diagnostic impressions of somatic dysfunction, and their correlation were evaluated using the $\chi^{2}$ test. The frequency of documentation was not significantly altered after viewing the videotape. A sequence of hospital-based instruction in osteopathic principles and practices has been initiated at more than 50 osteopathic institutions, and the problems related to continuing research of this type have emerged.

Supported by Burroughs Wellcome Osteopathic Research Fellowship No. F-89-07.

\section{Prostaglandin-second messenger interactions in the regulation of acidification in a model renal epithelium}

RAY D. PAGE, MS

THOMAS YORIO, PhD

Department of Pharmacology
Texas College of Osteopathic Medicine Fort Worth, Texas

Our laboratory recently demonstrated that prostaglandins (PGs), namely $\mathrm{PGF}_{2 \alpha}$, inhibited acidification in the skin of Rana pipiens, an $\mathrm{H}^{+}$-secreting epithelium ( $J$ Pharm Exp Ther 252:929). Prostaglandins appear to function to maintain a low basal $\mathrm{H}^{+}$excretion rate and to regulate intracellular $\mathrm{pH}$. The mechanisms by which PGs regulate these cellular processes is not understood. It may be via effects on second messenger function.

The purpose of this study was to determine if the activation of protein kinase $\mathrm{C}(\mathrm{PKC})$ alters $\mathrm{H}^{+}$ excretion in this tissue. The intact abdominal skin of the southern frog was used as a model epithelium. Paired skins were mounted in modified Ussing chambers and $\mathrm{H}^{+}$fluxes were determined. Mezerein, a PKC activator, was administered to the serosal media, and the tissue was allowed to flux under aeration for 120 minutes. Additional experiments were performed using phorbol myristate acetate (PMA) and $4 \alpha$-PMA, its inactive epimer, in the serosal media. $\mathrm{H}^{+}$secretion into each media was determined and reported as (nanomoles) $\left(\mathrm{cm}^{-2}\right)\left(\mathrm{hr}^{-1}\right)$. Mezerein $\left(10^{-8} \mathrm{M}\right)$ showed a significant decrease in mucosal and serosal $\mathrm{H}^{+}$excretion from 42 to 27 and 14 to -11 , respectively $(P<.01)$. Similar magnitudes of inhibition were observed with mezerein in the presence of ibuprofen $\left(10^{-5} \mathrm{M}\right)$. Phorbol myristate acetate produced a significant inhibition of both mucosal and serosal $\mathrm{H}^{+}$ excretion from 48 to 39 and -6 to -16 , respectively $(P<.05) .4 \mathrm{~L} \alpha$-PMA showed no significant change. Additional frogs received injections of ibuprofen $(10 \mathrm{mg} / \mathrm{kg})$ three times per day for 3 days. Cytosolic PKC activity was then assayed in the isolated "split" epithelium of the frog skin by measuring the incorporation of $\left[{ }^{32} \mathrm{P}\right]$ from $\left[{ }^{32} \mathrm{P}\right] \mathrm{ATP}$ into histone and was expressed as picomoles of ${ }^{32} \mathrm{P}$ transferred per milligram of protein per minute. Basal phosporylation rates in ibuprofen-treated animals were significantly less as compared with controls, 137 and 93, respectively. Stimulation of PKC activity was approximately 2.5 -fold in both groups and was reversed by $\mathrm{H} 7$, an inhibitor of PKC phosphorylation. Preliminary studies in tritiatedmyoinositol incubated tissues showed nonconclusive evidence of a $\mathrm{PGF}_{2 \alpha}$-mediated activation of the phosphoinositide (PI) pathway.

These data suggest that regulation of acidification processes and intracellular $\mathrm{pH}$ may be mediated through PKC activation. Activators of PKC exhibited the capability of inhibiting both the mu- 
cosal and serosal $\mathrm{H}^{+}$excretion mechanisms similar to that previously reported with $\mathrm{PGF}_{2 \alpha}$. However, further studies are required to determine if $\mathrm{PGF}_{2 \alpha}$ is indeed altering $\mathrm{PKC}$ activity through the PI cascade or another second messenger system.

Supported in part by Burroughs Wellcome Osteopathic Research Fellowship No. F-89-08 (R.P.) and the AHA, Texas Affiliate, Texas Research Enchancement Program (T.Y.).

\section{Preliminary observations of response in patients with cervical pain to osteopathic manipulative treatment}

\author{
EDWARD WALKO, DO \\ CHRISTINE JANOSEK, DO \\ Chicago College of Osteopathic Medicine \\ Chicago, Illinois
}

Patients currently under the care of the coinvestigator's practice were recruited and gave informed consent to participate in the preliminary study. This study evaluated the procedures and forms to be used in future clinical research. Five subjects with cervicothoracic pain participated in the study.

Patients had been receiving treatment for cervical pain for periods of 4 to 6 weeks. During the study, each subject had an initial visit and three subsequent weekly visits for treatment and data collection. Data collection included examination, use of pain scale to rate pain experience, response to a pain questionnaire, record of pain medication use, and a thermographic examination. Both investigators examined and recorded their findings related to the cervical pain, and the principal investigator administered osteopathic manipulative treatment during the first three visits based on the findings and assessment. Acceptable interexaminer agreement was obtained. A pain scale that was familiar to the patients reported pain on a scale of zero (no pain) to 10 (relative to the patient's experience of most severe pain). The average beginning scale of 5 decreased at each visit and averaged 3.4 at the final visit. The pain scale findings were supported by $67 \%$ positive responses to the questions related to decrease in pain experiences. Over the course of four thermographic measurements, there was an average decrease of $0.98^{\circ} \mathrm{C}$ in dorsal skin temperatures from the midthoracic level to the hair line.

The relationship between physicians' findings, pain measures, and thermographic analysis are cur- rently being analyzed. In the five cases, each patient reported some relief from cervical pain as evidenced on the pain scale and in response to the questions. Thermographic evidence, a decrease in elevated skin temperature in the affected area, greater than $0.3^{\circ} \mathrm{C}$, was considered significant and supportive of the subject response.

Supported by Burroughs Wellcome Osteopathic Research Fellowship No. F-89-11.

\section{Osteopathic manipulative treatment/osteopathic principles and practice}

\section{The role of osteopathic manipulation in the treatment of acute asthmatic patients: A pilot study}

\section{B. BERKOVICH, MS}

A. ALTER, DO

J. NOVOTNY, $\mathrm{PhD}$

University of New England College of Osteopathic Medicine

Biddeford, Maine

Pediatric patients experiencing mild to moderate asthmatic symptoms during an acute crisis can benefit from the use of trigger point and soft-tissue manipulation in addition to the standard practice of pharmacologic therapy.

A pediatric outpatient population, consisting of newly and previously diagnosed asthmatic subjects, was randomly divided into two groups. Both groups received aerosolized Ventolin (Glaxo) by either nebulizer or metered-dose inhaler. Prior to the pharmacologic intervention, members of the control group underwent therapeutic touch techniques. Those children not involved as controls experienced three forms of manipulation as follows: (1) trigger point therapy along the sternum, between the fifth and sixth ribs, (2) cervical fascial release with mild hyperextension of the cervical area, and (3) rib raising, a rhythmic stretching of the soft tissue along the thoracic spine. Three peak expiration flow rates were collected from each child, one before and one after manipulation or therapeutic touch and one after the administration of the bronchodilator. Ad- 
ditional signs such as wheezing, oxygen saturation, respiratory rate, intercostal retractions, nasal flaring, and prior medications were also evaluated. All children were followed up at the end of 1 week and reevaluated for continuing signs and symptoms of the disease. Parents kept diaries detailing the child's progress.

Those children receiving osteopathic manipulative treatment (OMT) fell into three categories as follows: (1) favorable response to the OMT, decreasing the amount of medical intervention needed, (2) enhancing the standard bronchodilator therapy, and (3) no benefit in treating the asthmatic crisis.

Supported in part by the Family Practice Grant Fellowship and the Wilbur V. Cole, Sr, DO, Research Fellowship.

\section{The role of respiration in the tension production of myofascial tissues}

\author{
JAMES D. CUMMINGS, DO \\ JOHN N. HOWELL, PhD \\ Ohio University College of Osteopathic Medicine \\ Athens, Ohio
}

Respiration is commonly used as an intrinsic force by practitioners of osteopathic manipulation. Although widely accepted as effective, little has been written to show objective measurements of the role of respiration in tension production by myofascial tissues. Data from the Somatic Dysfunction Laboratory at Ohio University College of Osteopathic Medicine, Athens, documents the impact of respiratory mechanics on the tension produced by resting myofascial tissues of the human elbow flexor mechanisms. Torque measurements obtained from digitally recorded strain gauge output clearly demonstrate the mechanical impact of the respiratory cycle on resting myofascial tissue tension. Additionally, a neurologically induced influence has been reported by Kisselkova and Georgiev ( $J$ Appl Physiol 1979;46:1093-1095). They reported resting electromyographic activity from the biceps brachii, quadriceps femoris, and gastrocnemius muscles cycled with respiration following bicycle ergometer exercise, thus demonstrating that nonrespiratory muscles receive input from the respiratory centers. These studies document both a mechanically and a neurologically mediated influence on the tension produced by myofascial tissues. This gives objective verification of the clinically observed influence of respiration on the musculoskeletal system and validation of its potential role in osteopathic manipulative treatment.

Supported by AOA Bureau of Research grant No. 89-08259.

\section{Osteopathic manipulative treatment clinical research in a private practice setting: Lessons learned in a pilot study}

\author{
STEPHEN D. HUSBAND, PhD \\ JEROME J. PLATT, PhD \\ ELEANOR MASTERSON, DO \\ University of Medicine and Dentistry of New Jersey- \\ School of Osteopathic Medicine \\ Camden, New Jersey
}

This presentation will address some of the perils and pitfalls in conducting "real-life" clinical research with osteopathic manipulative treatment (OMT) in a private practice setting. In a pilot study intended to relate the efficacy of OMT for patients with back pain to patient demographic or psychological variables (or both), such as depression, perception of pain, and hopelessness, a number of problems were encountered that eventually confounded completion of the study as it was originally envisioned.

The purpose of this presentation is to discuss some of the problems inherent in conducting OMT research, as well as highlighting strategies for avoiding or overcoming problem areas using our experiences with the pilot study to illustrate points. Some of the potential problem areas that may be encountered by the OMT researcher include the following:

1. Subject selection criteria such as volunteer bias, self-selection, impact of informed consent, and the like. For example, in our pilot study patients did not volunteer to participate for a number of reasons; these reasons will be discussed along with ways to minimize this kind of problem.

2. Patient-related variables such as "positive malingering," insurance coverage status, litigation or worker's compensation status, psychological status, prior experience with and expectations of OMT, and referral source.

3. Environmental variables such as demand characteristics of the office setting, degree of confidentiality of data collected (both real and perceived), the use of psychologically oriented instruments in a physical medicine setting, and the time required for subjects to complete the instruments. 
4. Research management issues such as researcher-clinician interaction, control of the data collection process, difficulties in collecting data in a private office setting, the need for on-site research assistants and management of the assistants, and the way in which the research is presented when recruiting subjects from patients in a private practice office.

\section{Incidence of somatic dysfunction in a random population of school teachers attending the Fort Worth, Texas, ISD health fair}

SHEILA D. PAGE, BS

J. DICKEY, DO

RICHARD BALDWIN, DO

TIM WRIGHT, BA

Texas College of Osteopathic Medicine

Fort Worth, Texas

The field of osteopathic medicine has very few baseline studies defining the incidence of somatic dysfunction in the general population and its relevance to the incidence of specific disease states. The purpose of this study was to present this type of information on a random group of people who are not actively seeking healthcare.

The individuals screened were teachers attending the Fort Worth (Texas) Independent School District health fair who submitted to a structural evaluation. The examination was performed on 68 persons with a mean age of 38.6 years (range, 22 to 70 years), with 58 females and 10 males. The examiners did not have access to the information on the questionnaire before the screening, nor did they ask questions during the examination. The examination included evaluation of femoral heights, standing flexion test, and palpation of the axial spine for asymmetry, restriction of motion, and tissue texture change (ART).

The highest percentage of somatic dysfunction in this group occurred in vertebrae C2 (44\%), C7 through $\mathrm{T} 1$ ( $35 \%$ to $40 \%$ ), T5 through $\mathrm{T} 7$ ( $38 \%$ to $44 \%$ ), and T12 through L2 (37\% to $40 \%$ ). The lowest incidence occurred at C1 (13\%), T9 through T11 (16\% to $20 \%$ ), and L5 (18\%). The total number of patients reporting a history of urinary tract infection was 15 , of which $11(73 \%)$ had somatic dysfunction at T12 through L2. In addition, $12(18 \%)$ of the total persons screened reported having peptic ulcer disease or heartburn. Of these, $58 \%$ had somatic dysfunction in the T5 through T9 region. The total number of persons with somatic dysfunction in this region was $47(69 \%)$.

These data suggest that somatic dysfunction occurs most often in transitional areas of the spine. They also suggest that certain disease processes are present concurrently with somatic dysfunction in specific vertebral sections. Somatic dysfunction of T5 through T9 was common in this group, and prospective studies should be done to determine whether ART changes in the T5 through T9 region is an early indicator of peptic ulcer disease in an otherwise asymptomatic person.

Supported in part by an Undergraduate Teaching Fellowship (S.P., T.W.).

\section{Athletic functional demand and posture}

\author{
MICHAEL A. KUCHERA, DO \\ MICHAEL BEMBEN, PhD \\ WILLIAM A. KUCHERA, DO \\ FONTAINE PIPER, PhD \\ Kirksville College of Osteopathic Medicine \\ and \\ Northeast Missouri State University \\ Kirksville, Missouri
}

Structure-function relationships often provide important clinical perspectives. This study examined the relationship between the high functional demands of athletic activities, posture, and injury. A history of trauma, pain, and athletic activity was obtained from 182 volunteer collegiate underclassmen. Anthropomorphic measurements, postural radiographic series, palpatory structural examinations, and photographic center-of-gravity analyses were performed on all subjects. Historical data, including previous functional demand levels in each of the cardinal planes of motion, were converted using predetermined constants to a form that could be entered into a computer spreadsheet along with measured postural and anthropomorphic data. Use of underclassmen not engaged in collegiate sports failed to yield the postulated control group because many had at least as much precollegiate athletic history as those in the sports group. Statistical analysis was therefore performed on subgroups of the total population according to the criteria of the specific question.

Subjects reporting a significant injury history or tight psoas muscles were found to have higher incidences of noncompensated fascial patterning 
(Zink). Also, a golf team-specific palpatory finding of rotation to the right on the right oblique sacral axis within Zink's otherwise commonly compensated fascial pattern was frequently noted. In subjects with at least moderate back pain within the last year, there was higher incidence of noncompensated patterning while during the same period subjects with compensated patterns were more likely to have had no back pain.

There was no significant relationship between total previous functional demand and any radiographic postural variable, but previous functional demand in the sagittal plane did appear to be associated with a higher incidence of radiographically anterior L3 weight-bearing lines. Center-of-gravity measurements did not correlate with any radiographic variables. The total number of radiographically demonstrated postural abnormalities was not related to the history of back pain, but patients with a history of back pain had a higher radiographic incidence of elevated pelvic index (Jungmann).

While too few collegiate injuries occurred to examine correlations with preexisting postural previous functional demand, this study documents structure-function relationships involving injury and pain histories with the palpatory structural examination.

Supported by AOA Bureau of Research grant No. 87-04227.

\section{Redesign and construction of a device for objective measurement of segmental autonomic changes in man}

\author{
MICHAEL L. KUCHERA, DO \\ EMIL BLACKORBY \\ JOHN HEARD, $\mathrm{PhD}$ \\ Kirksville College of Osteopathic Medicine \\ Kirksville, Missouri
}

One of the most significant research periods in the osteopathic profession took place at the Kirksville College of Osteopathic Medicine between 1951 and 1966. During this time, under the direction of I. M. Korr, $\mathrm{PhD}$, and associates, research data were accumulated that formed the basis for the facilitated segment concept, which is one of the cornerstones of the modern osteopathic approach to patient care. Objective evidence of segmental autonomic change was recorded by a series of devices that measured reliable, reproducible, and readily explained objective physiologic parameters, including electrical skin resistance. The data positively correlated with clinical postural, palpatory, and historical findings. Because neither of Korr's two original electrical skin resistance machines is now functional, and their original design diagrams were unwittingly destroyed, continuation of this important research was seriously hampered.

This project has combined the original design engineer (Blackorby) with current electronic and computer technology resulting in a redesigned prototypal instrument capable of measuring electrical skin resistance. Components not commercially available were built in-house to permit measurement of electrical skin resistance through 23 sequentially sampled probes arranged along an $X$ axis. The probes are free to move topographically along the $Y$ axis at a fixed rate to allow analysis of the entire back in less than 3 minutes. The analog input signal is then converted into a digital signal that the computer can process, and each electrical skin resistance value is assigned to the $(X, Y)$ coordinates of specific locations anatomically and physiologically related to the sympathetic outflow of the thoracolumbar regions.

After calibration trials, the electrical skin resistance data collection instrument should permit objective quantification useful for research analysis seeking to correlate segmental autonomic findings in viscerosomatic and somatovisceral conditions, and may provide a means of evaluating the effect of treatments purported to modify segmental somatic dysfunction.

Supported by the Warner Research Fund of Kirksville College of Osteopathic Medicine and by the National Levitor Center.

\section{Treatment options in fibromyalgia syndrome}

\author{
B. R. RUBIN, DO \\ R. G. GAMBER, DO \\ C. A. CORTEZ, RN \\ T. J. WRIGHT \\ J. SHORES, PhD \\ G. DAVIS, RN \\ Texas College of Osteopathic Medicine \\ Fort Worth, Texas
}

Fibromyalgia syndrome is characterized by chronic musculoskeletal pain and palpable tenderness at specific anatomic sites. This study examined the 
effects of drug and manipulative treatment on tenderness and the patients' perception of quality of life.

In this double-blind study, 37 patients with fibromyalgia syndrome were randomly assigned to one of four treatment groups. They received the following: (1) ibuprofen, alprazolam, and manipulation, or (2) a placebo and manipulation, or (3) ibuprofen and alprazolam, or (4) a placebo alone. The treatment lasted 6 weeks. On first contact and at 2 -week intervals thereafter, dolorimeter readings were taken at each of 11 bilateral sites and a five-item quality-of-life scale was completed.

Multiple analysis of variance was used to test the effects of treatment over time. The treatment groups were found to be significantly different $(P<.05)$ from one another. This effect was independent of time. Sheffe contrasts confirmed that drug therapy alone resulted in significantly less tenderness than did drugs with manipulation, a placebo with manipulation, or a placebo alone. Further, patients who received a placebo with manipulation reported significantly lower levels of fatigue.

The findings of this study confirm the effectiveness of drug therapy in the treatment of fibromyalgia syndrome. The findings mitigate against the use of manipulation alone in the treatment of this syndrome. The study suggests that drug treatment with manipulation is slightly more effective than a placebo with manipulation or a placebo alone. When drug therapy is administered with manipulation, the patient's quality of life is improved.

Supported by AOA Bureau of Research grant No. 88-11282.

\section{Somatic and psychosocial comparisons in cardiac, noncardiac, and normal subjects: Somatic findings}

\author{
JAMES A. THESING, BS \\ DANIEL J. KRANITZ, BA \\ ANTHONY G. CHILA, DO \\ Ohio University College of Osteopathic Medicine \\ Athens, Ohio
}

This study sought to explore somatic differences between three groups of subjects: those who had sustained an acute myocardial infarction, those who had been receiving osteopathic manipulative treatment for noncardiac diagnoses, and "normal" subjects. A limited osteopathic structural exami- nation was performed that emphasized alteration of tissue texture and mechanical range of motion in the cervical column, thoracic column, and costal cage. Manipulative interventions were not part of this study.

Twenty-four subjects were enrolled in this study: 4 after suffering a myocardial infarction, 12 after manipulative treatment, and 8 "normal"subjects. The palpatory examinations used were as follows: translatory motion testing of the cervical column, anterior compression testing of the thoracic column, respiratory rib excursion, and paraspinal cutaneous reactivity. All palpatory assessments were done by student physicians specifically trained in the examination format. Subjects were randomly allocated at the time the examinations were performed. The examiners alternated with each other in the examination of each subject. It was hypothesized that there would be differences between groups in somatic findings.

A relatively high agreement on somatic findings was achieved between examiners. Certain areas of the spinal complex presented apparent difficulty in achieving palpatory agreement. No statistically significant differences were noted between the groups.

Supported by the Norman J. Larson Clinical Research Fellowship, 1989. Funding was provided by the Norman J. Larson Clinical Research Fund.

\section{Results of computer evaluation of osteopathic manipulative treatment by pressure transducers (forced displacement meters) and electromyographic data}

\author{
CHARLES STEINER, DO \\ N. GUZELSU, PhD \\ C. BOLECEK, PhD \\ University of Medicine and Dentistry of New Jersey- \\ School of Osteopathic Medicine \\ and \\ Biomedical Engineering Department \\ Rutgers University \\ Piscataway, New Jersey
}

This study developed a technique that measured the effectiveness of osteopathic manipulative treatment of low-back pain by quantification of change in pain and integrated electromyographic data.

The integrated electromyographic data were obtained from bilateral muscle sites at the L3-L4 (lum- 
bar) spinal level by surface electrodes during a 10second standing isometric resistance of flexion task, as described by Schultz et al. The electrodes are placed on the skin surface adjacent to the multifidus, longissimus rectus abdominis, and lateral oblique muscles. The integrated electromyographic data were measured for five external load levels ( 0 to $5.0 \mathrm{~kg}$ ) with 1 -minute rest periods between loads. Measuring the change in integrated electromyographic data with force generated (due to an external load) enabled us to characterize the muscle efficiency of electrical activity. Patients were selected for: (1) the presence of lumbar pain of muscular origin, and (2) the absence of progressive neurologic changes, weakened or loss of reflexes, atrophy of tissues supplied by derivatives of L5-S1 nerve roots, systemic disorders, such as tumors, diabetic neuropathy, visceral pathologic findings, and spinal fractures.

Statistical analysis (using analysis of variance) of the control group total erector spinae (ie, right and left side multifidus plus longissimus rectus abdominis) integrated electromyographic slope data showed no significant change between sessions. However, the patient group with total erector spinae integrated electromyographic slope $(P<.02)$ and pain data did show a significant treatment effect between before and after session 1 . These results suggest that the treatment of low back pain of muscular origin, with osteopathic manipulative treatment, will result in a decrease in pain and integrated electromyographic slope and thus, an increased muscle efficiency of electrical activity. The beneficial effects of osteopathic manipulative treatment carried through four sessions.

Supported by University of Medicine and Dentistry of New Jersey Foundation grant No. 8-88 and AOA Bureau of Research grant No. 88-06-204.
Biotherapeutics

Arlington (Texas) Public Health Department and

Texas College of Osteopathic Medicine

Fort Worth, Texas

Because of their clinical significance in the management of the acquired immunodeficiency syndrome (AIDS), Mycobacterium avium infections were investigated in individuals who were human immunodeficiency virus (HIV) negative in an attempt to correlate immunologic function with the presence of these opportunistic pathogens in a nonAIDS patient population. Peripheral blood lymphocytes were assayed for their ability to react to phytohemagglutinin, pokeweed mitogen, and Staphylococcus A. Flow cytometry was used to quantitate various lymphocyte populations by using fluoroisothiocyanate-labeled monoclonal antibodies directed to B1, B4, CD3, CD4, and CD8 epitopes. Infection with $M$ avium was confirmed by biochemical assays of repetitive culture isolates and thin-layer chromatographic analysis of lipids extracted from isolates cultivated in Middlebrook 7H9 and supplemented with OADC. All except one of the cultures exhibited smooth-colony morphology, either the transparent or dome-opaque variety, and contained the serovar-specific glycopeptidolipid antigens associated with the $M$ avium complex. Although all of the patients exhibited a normal blastogenic response to mitogens, several exhibited a reduced $\mathrm{CD} 4 / \mathrm{CD} 8$ ratio, similar to that observed in patients with AIDS. Serum was examined for the presence of the GPL antigens; however, none were observed at levels detectable by thin-layer chromatography. Results suggest that chronic $M$ avium infections may alter immunologic functions in some patients by mechanisms not yet determined.

Supported by AOA Bureau of Research grant No. 88-11-285and the National Institutes of Health, Bethesda, Md.

\section{The effect of fluoride on the myocardium}

\section{Immunologic functions of patients infected with Mycobacterium avium}

W. W. BARROW, PhD

S. K. TASSELL, MS

B. KING, RN

J. W. MEASEL, PhD

S. E. WEIS, DO
C. H. GREENE, PhD

D. A. DeBIAS, $\mathrm{PhD}$

D. A. DeBIAS, MD

W. L. YOUNG, AA

C. V. DIMITRIU, MS

Department of Physiology and Pharmacology

Philadelphia College of Osteopathic Medicine

Philadelphia, Pennsylvania 
Enzymatically isolated canine ventricular myocytes maintained in solution metabolize anaerobically similarly to the ischemic areas of the intact myocardium and the transplanted heart. They can be maintained in a viable state without differentiation for hours, permitting incubation with various substrates. Fluoride is an inhibitor of several glycolytic enzymes. It is a natural constituent of drinking water in many areas, and also a frequent additive to water and many consumer products. Direct effects of fluoride on glycolytic pathways were examined using isolated canine ventricular myocytes obtained from healthy hearts.

Cells were incubated with and without various concentrations of fluoride and glucose substrate plus insulin for $0,30,60$, and 90 minutes. Cells were evaluated structurally by morphometric analysis of electron micrographs and metabolically by measurement of total adenosine triphosphate content, lactic and pyruvic acid production, and glucose uptake. Structural analyses were correlated to metabolic activity.

\section{Hemoglobin A1c as a screening test for diabetes mellitus in the elderly}

\author{
H. TIMOTHY DOMBROWSKI, DO \\ THEODORE J. PASSON, JR, PhD \\ ALICE PERKINS, RN, GNP \\ University of Medicine and Dentistry of New Jersey- \\ School of Osteopathic Medicine \\ Stratford, New Jersey
}

The project objectives were: (1) to study the utility of the hemoglobin $\mathrm{A} 1 \mathrm{c}(\mathrm{HbA} 1 \mathrm{c})$ test as a screening test for diabetes mellitus in the elderly and, (2) to establish the range of abnormal $\mathrm{HbA} 1 \mathrm{c}$ values that correlate with a diabetic response on a 2 -hour oral glucose tolerance test.

Twenty-five elderly subjects (aged 65 to 81 years) entered the study and had a blood specimen drawn for fasting blood sugar and HbA1c tests. Fourteen subjects were then assigned to one of two groups based on whether the $\mathrm{HbA1c}$ concentration was normal or abnormal and underwent a 75-g, 2-hour oral glucose tolerance test. The results of the oral glucose tolerance tests were classified according to the National Diabetes Data Group and correlated with the $\mathrm{HbA1c}$ values, using a two-tailed test to derive a $P$ value.

Eighteen of 25 elderly subjects had both fasting blood sugar and $\mathrm{HbA1c}$ values within the normal range. The remaining seven had normal fasting blood sugar test results, but elevated $\mathrm{HbA1c}$ levels. Seven subjects with normal $\mathrm{HbA} 1 \mathrm{c}$ values and seven subjects with elevated $\mathrm{HbA} 1 \mathrm{c}$ values underwent a standard 2-hour oral glucose tolerance test. Only three of the seven subjects with elevated $\mathrm{HbA} 1 \mathrm{c}$ values had a diabetic response on the oral glucose tolerance test.

The three subjects with a diabetic oral glucose tolerance test response had $\mathrm{HbA1c}$ values greater than $7 \%$, while all of the other subjects had values less than $7 \%$. The HbA1c levels greater than $7 \%$ were five standard deviations above the normal mean, and when these $\mathrm{HbA1c}$ levels were compared with the levels in the group with normal oral glucose tolerance test results, they were significantly different $(P=.001)$.

The results of the present study suggest that $\mathrm{HbA1c}$ values can be divided into three categories: normal levels within two standard deviations with normal oral glucose tolerance test responses, intermediate levels between the second and fifth standard deviation with varying oral glucose tolerance test responses, and abnormal levels that are greater than or equal to five standard deviations and are always associated with a diabetic response to an oral glucose tolerance test. If further study supports these suggestions, oral glucose tolerance tests would only be necessary for patients with $\mathrm{HbA1c}$ levels within the intermediate range.

\section{The use of choline magnesium trisalicylate in the treatment of chronic low-back pain}

\author{
ROBERT LEVINE, MD \\ SUSAN MONTPETIT, PharmD \\ JOHN DOOLEY, PhD \\ ROBERT GRANDY, MS \\ Gertrude Levin Pain Clinic \\ Harper Hospital \\ Detroit, Michigan
}

This double-blind crossover study determined the effectiveness of choline magnesium trisalicylate (Trilisate) in the treatment of chronic low-back pain.

All patients had low-back pain for longer than 6 months. Individuals with a neurologic defect or a significant psychologic abnormality were disqualified from entry into the study. Subjects were obtained from pain clinic referrals or responses to 
public service announcements. Patients were randomly assigned to a placebo or active medication group and crossed over at 6 weeks. All patients completed a pain and activity questionnaire daily. All patients were reevaluated after each segment. Of the 99 patients who qualified for entry into the study, 76 had usable data.

There were psychological differences between the groups. Patients with chronic back pain obtained from responses to public service announcements had Minnesota Multiphasic Personality Inventory test results within normal limits. Patients with back pain who were referred to the clinic had the classic pain "conversion V" on their Minnesota Multiphasic Personality Inventory test. The Sickness Impact Profile of patients referred for treatment showed a greater overall level of health activity impairment than those seen solely for the study.

Compared with the placebo, while taking active medication, pain decreased and activity increased. During the first 6 -week segment, $72 \%$ of the patients receiving choline magnesium trisalicylate had mild to excellent relief, and $22 \%$ who were taking placebo had mild or good relief. At 6 weeks, no pain relief was reported in $28 \%$ of the patients receiving choline magnesium trisalicylate or in $78 \%$ of the patients receiving placebo $(P=.0003)$. During the second 6-week period, there was mild or good relief in $68 \%$ of those receiving active medication and in $29 \%$ of those using placebo. No pain relief was reported during the second 6 weeks in $35 \%$ of the patients receiving the active drug or in $71 \%$ of the patients receiving placebo $(P=.005)$.

It is concluded that choline magnesium trisalicylate is effective in the management of chronic low-back pain.

Funding for this project was obtained from The Purdue Frederick Company.

\section{Genes located on extrachromosomal plasmids in Staphylococcus aureus influence borderline oxacillin resistance}

J. K. PIERCE, $\mathrm{PhD}$

\section{A. ANDERSON \\ DAVID WELCH, $\mathrm{PhD}$}

Kirksville College of Osteopathic Medicine

Kirksville Missouri

and

The University of Oklahoma Health Science Center

Oklahoma City, Oklahoma
Staphylococcus aureus is a normal inhabitant of human skin surfaces, and is responsible for localized skin infections, endocarditis, and septicemia. Infections due to methicillin- or oxacillin-resistant $S$ aureus are a significant problem in this country. Strains that possess minimum inhibitory concentrations for oxacillin of 1 to $4 \mu \mathrm{g} / \mathrm{mL}$ have been termed borderline or intermediate resistant. We assayed clinical isolates of $S$ aureus for chromosomal plasmids. Borderline-resistant strains were cured of plasmids by growth in media containing ethidium bromide. These strains were found to have an increased sensitivity to oxacillin, penicillin, ampicillin, and amoxicillin. These results indicate that the probable location for genes responsible for borderline oxacillin resistance is on plasmids contained in these strains. The results presented compare the plasmids found in these strains with respect to size and restriction enzyme profiles.

\section{A retrospective study of abdominal distress in 51 Asian immigrants}

\author{
MAURICE A. RAMIREZ, BS \\ DAVID VISOKEY, BS \\ DAVID PLUNDO, DO \\ NANCY AKINS, DO \\ University of Osteopathic Medicine and \\ Health Sciences College of Osteopathic Medicine \\ Des Moines, Iowa
}

This study reviewed the records of 51 Asian immigrants to the United States. The immigrants were divided into two groups. Group A subjects had intestinal parasitosis as diagnosed by direct microscopy. Group B subjects had no intestinal parasitosis. The subjects were followed up for periods of no less than 90 days. In group A, four subjects were seen at the clinic because of abdominal pain. Of these four subjects, one had a fecal impaction and three had idiopathic abdominal distress that was self-limiting. In group B, four subjects had abdominal pain. One had gastritis, one had constipation, one had mild gastric reflux, and one had an idiopathic condition that was self-limited. Eight patients in Group A and seven in Group B were unavailable for follow up. There was no statistically significant difference in the incidence of abdominal distress between group A and group B $(P>.10)$. Idiopathic abdominal distress was more common in group $\mathrm{A}$ than in group $\mathrm{B}(P<.001)$. Large-scale studies are required to conclusively determine the 
relative incidence of postparasitic abdominal distress.

Research funded by the University of Osteopathic Medicine and Health Sciences Tower Family Practice Department.

\section{The wound-healing effect of povidone iodine cream on experimentally induced wounds}

ROBERT P. GRANDY, MS

CELINE M. STAHL-BAYLISS, MD

The Purdue Frederick Company

Norwalk, Connecticut

Povidone iodine is frequently applied to wounds as a topical antiseptic. Although some have reported that povidone iodine may interfere with wound healing, others report that povidone iodine has no deleterious effect on wound healing, and even enhances wound healing by controlling infection. The majority cited the fact that povidone iodine adversely affecting wound healing is based on in vitro and animal models and not clinical evaluation. These models have involved the use of a povidone iodine surgical scrub or skin cleanser that has been recognized as deleterious to wound healing because of the detergent component in each of these formulations.

To properly evaluate the effect of povidone iodine microbicides on wound healing, we used the following in vivo human model. Uniform clinical blisters were produced on the volar aspect of the forearm of 12 volunteers with $50 \%$ ammonium hydroxide and unroofed after 15 minutes. The wound produced is equivalent to a superficial abrasion or second-degree burn. To simulate infection, each wound was inoculated with $1 \times 10^{5}$ Staphylococcus aureus 24 hours later. The wounds were occluded with a dressing for 4 hours, the dressing was removed, and the wounds were treated with $0.1 \mathrm{~mL}$ of either $5 \%$ povidone iodine cream or polymyxin B sulfate-neomycin sulfate cream for 10 minutes; one control wound received no treatment. The test products were then rinsed from the wound with sterile water and culture samples were taken. Each wound was treated twice daily until healed.
Evaluation of epithelialization was performed with a stereomicroscope. Both products significantly $(P<.01)$ reduced the number of viable $S$ aureus by 2 logs compared with the control. Povidone iodine cream $(5 \%)$ significantly $(P<.05)$ shortened wound healing time compared with the control. Polymyxin B sulfate-neomycin sulfate cream showed no difference $(P>.23)$ in time to wound healing compared with the control. Povidone iodine cream (5\%) showed substantially broader microbicidal activity than polymyxin B sulfate-neomycin sulfate in in vitro testing. We conclude that $5 \%$ povidone iodine cream is an effective topical antiseptic agent and appears to enhance wound healing compared with either no treatment or polymyxin B sulfate-neomycin sulfate in this in vivo human infected-wound model.

\section{The diagnosis of fetal anterior abdominal wall defects using ultrasonography: A case study}

\section{BRADLEY F. SCHWARTZ, DO}

Texas College of Osteopathic Medicine

Fort Worth, Texas

Omphalocele and gastroschisis are defects in the anterior abdominal wall through which abdominovisceral organs protrude. The prenatal diagnosis of these two clinical entities can usually be made by using pelvic ultrasonography. Furthermore, the two defects can be differentiated during a routine prenatal obstetrical check-up by utilization of ultrasonography. The incidence of omphalocele has been reported to be anywhere from 1:1400 to $1: 10,000$. The incidence of gastroschisis is more rare, and occurs in approximately $1: 12,000$ pregnancies.

We report a case of omphalocele detected by ultrasonography in the 20 th week of gestation measured from the first day of the last menstrual period, during a routine prenatal obstetrical checkup.

Funding and major research supported by Frederick M. Wilkins, DO, Chairman, Department of Radiology, Texas College of Osteopathic Medicine, and Robert L. Nelson, DO, Chairman, Radiology Department, Dallas-Fort Worth Medical Center. 